Gerald H. Ristow

\title{
Pattern Formation in Granular Materials
}

With a Foreword by Siegfried Großmann and 83 Figures 


\section{Contents}

1. Introduction $\ldots \ldots \ldots \ldots \ldots \ldots \ldots \ldots \ldots \ldots \ldots \ldots \ldots \ldots$

2. Some Experimental Phenomena of Granular Materials... . 5

2.1 Shear flow ........................... 5

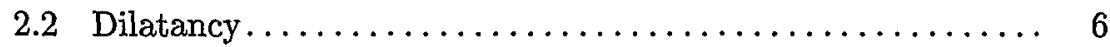

2.3 Solid-Fluid Transition $\ldots \ldots \ldots \ldots \ldots \ldots \ldots \ldots \ldots \ldots \ldots \ldots$

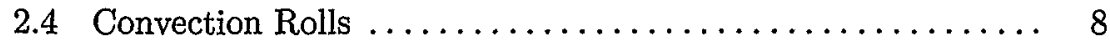

2.5 Free-Surface Flow $\ldots \ldots \ldots \ldots \ldots \ldots \ldots \ldots \ldots \ldots \ldots \ldots \ldots$

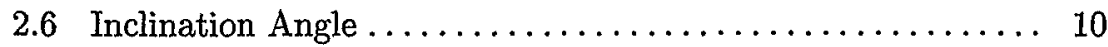

2.7 Density and Stress Fluctuations $\ldots \ldots \ldots \ldots \ldots \ldots \ldots \ldots \ldots \ldots$

2.8 Commonly Used Materials . . . . . . . . . . . . . 11

3. Vertical Shaking $\ldots \ldots \ldots \ldots \ldots \ldots \ldots \ldots \ldots \ldots \ldots \ldots \ldots$

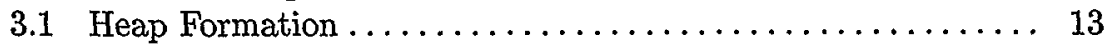

3.2 Convective Motion. . . . . . . . . . . . . . . . . 17

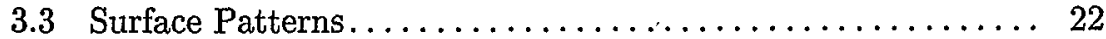

3.3.1 Surface Waves ...................... 23

3.3 .2 Stationary Patterns................... 24

3.4 Compactification and Clustering............... 29

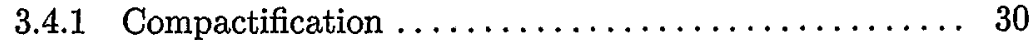

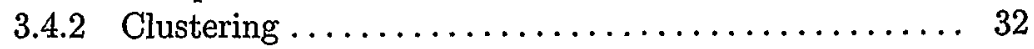

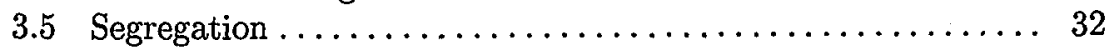

4. Horizontal Shaking $\ldots \ldots \ldots \ldots \ldots \ldots \ldots \ldots \ldots \ldots \ldots \ldots$

4.1 Solid-Fluid Transition $\ldots \ldots \ldots \ldots \ldots \ldots \ldots \ldots \ldots \ldots \ldots \ldots \ldots \ldots$

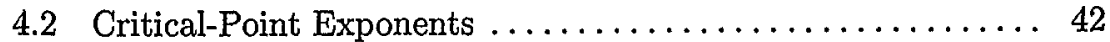

4.3 Crystallization $\ldots \ldots \ldots \ldots \ldots \ldots \ldots \ldots \ldots \ldots \ldots \ldots \ldots \ldots \ldots$

4.4 Convection Rolls $\ldots \ldots \ldots \ldots \ldots \ldots \ldots \ldots \ldots \ldots \ldots \ldots \ldots 45$

4.5 Surface Patterns........................ 46

4.6 Lifting the Hysteresis by Gas Flow .............. 48

4.7 Inverted Funnel Flow in Hoppers . . . . . . . . . . . 49 


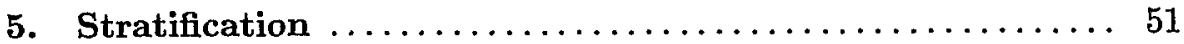

5.1 Experimental Findings $\ldots \ldots \ldots \ldots \ldots \ldots \ldots \ldots \ldots \ldots \ldots \ldots . \ldots 1$

5.2 Discrete Models ....................... 57

5.2 .1 Model Based on Angle of Repose ............ 57

5.2.2 Model Based on Energy Dissipation .......... 58

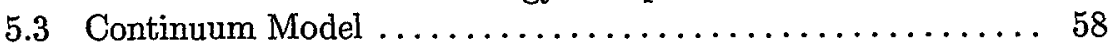

5.3 .1 Model Description ................... 58

5.3 .2 Steady-State Profiles.................... 60

5.3 .3 Stability Analysis $\ldots \ldots \ldots \ldots \ldots \ldots \ldots \ldots \ldots \ldots \ldots 6$

5.3 .4 Thin-Flow Regime................... 62

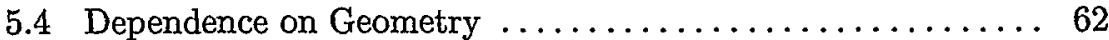

6. Conical Hopper........................ 67

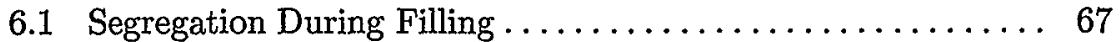

6.2 Static Wall Stresses ........................ 68

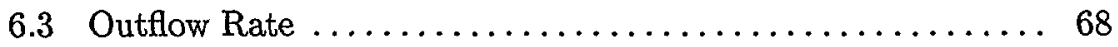

6.3.1 Dependence on Orifice ................. 69

6.3 .2 Dependence on Silo Geometry ............. 70

6.4 Flow Regions ........................ 71

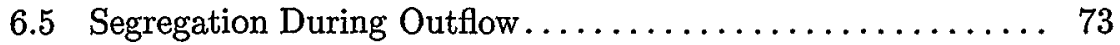

6.6 Density Waves .......................... 74

6.7 Dynamic Wall Stresses .................... 77

6.8 Silo Design to Decrease the Stress Fluctuations. . . . . . . . 79

7. Rotating Drum ........................... 81

7.1 Different Flow Regimes..................... 81

7.1 .1 Avalanches ...................... 82

7.1 .2 Continuous Surface Flow ................ 85

7.1 .3 Centrifugal Regime ................. 88

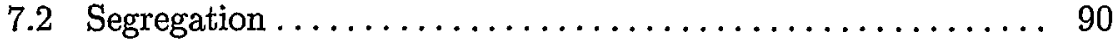

7.2 .1 Radial Size Segregation ................. 91

7.2 .2 Radial Density Segregation ................ 96

7.2.3 Interplay of Size and Density Segregation.......... 99

7.2 .4 Friction-Induced Segregation ................... 99

7.2 .5 End-Longitudinal Segregation ............... 101

7.2 .6 Axial Segregation . . . . . . . . . . . . . . . 102

7.3 Axial Band and Wave Dynamics ................. 105

7.4 Competition of Mixing and Radial Segregation .......... 107

7.5 Front Propagation and Radial Segregation . . . . . . . . . . 111

7.5.1 Experimental Setup and Studies.............. 112

7.5.2 Approximation Through Diffusion Process ........ . . 115

7.5.3 Concentration-Dependent Diffusion Coefficient ....... 116

7.5.4 Calculation of Diffusion Coefficients ............. 119

7.5.5 Front Propagation with and Without Segregation .... 123 
8. Concluding Remarks and Outlook ............. 125

A. Numerical Methods Used to Study Granular Materials . . 129

A.1 Monte Carlo Method........................ 129

A.2 Diffusing-Void Model . . . . . . . . . . . . . . . 130

A.3 Method of Steepest Descent.................. 130

A.4 Cellular Automata ...................... 131

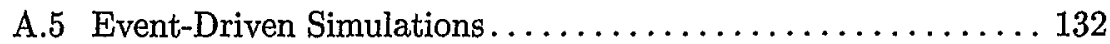

A.6 Time-Driven Simulations (Molecular Dynamics) . . . . . . . 133

A.6.1 Time-Integration Scheme . . . . . . . . . . . . 133

A.6.2 Forces During Collisions . . . . . . . . . . . . 134

A.6.3 Numerical Stability . . . . . . . . . . . . . . . . 140

A.6.4 Comparison with Experiments . . . . . . . . . . 141

A.7 Summary ........................... 144

References .............................. 145

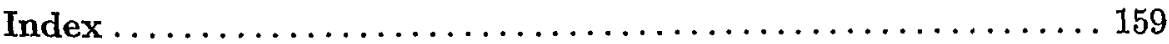

